Interactive comment on "Simulating precipitation decline under a Mediterranean deciduous Oak forest: effects on isoprene seasonal emissions and predictions under climatic scenarios" by Anne-Cyrielle Genard-Zielinski et al.

Anne-Cyrielle Genard-Zielinski et al.

christophe.boissard@lsce.ipsl.fr

Received and published: 12 July 2017

Referee \#1

General comments

None

Detailed comments

We do thank Referee \#1 for his/her comments. 
We do agree with all comments / changes suggested and will do the appropriate changes, but:

- Abstract:

Lin 20-23: the lowest ER values in October and April were 6 and $<2 \mu \mathrm{g}$ gDW-1 h-1 respectively

Lin 20: no, the lowest Gw values were observed between July and September $(<20$ moleH2O m-2 s-1) not in April (figure 2b)

Line 22: we do not understand this point; emission rates ER (measured values) are different from emission factors (ER normalized to temperature and PAR)

- Materials and Methods:

Pg 5 Lin 10: sampling volume varies between 0.45 and $0.9 \mathrm{~L}$ depending on the season and, thus, the expected emission intensity.

$\mathrm{Pg} 8$ Lin 7: indeed Netral site web is no more available and we do not have another web link.

- Results \& Discussion:

Pg 10 Lin 20: Electric resistivity tomography measurements have shown the heterogeneity of the karstic substrate organized as soil pockets developed between limestone rocks. Water and nutrient pools and dynamics probably differ greatly between the shallow upper soil layers and the soil pockets developed between limestone rocks. However, the soil trenches carried out in the site have shown that a calcareous slab often developed at a depth of $10-20 \mathrm{~cm}$ and that the roots of the oaks were rather distributed in this superficial humiferous horizon, and that only a few large roots cross this slab.

Pg 10, Lin 26: Consider the senescence of leaves: although senescence had just begun during this sampling period, but we did check that the enclosed branches were not senescent during our measurements. 
- Figure caption:

The same colors of the legends in all graphs: we used blue and red colors for ND and $A D$ respectively in all graphs; we are not quite sure what the referee wants.

Fig 1: in Fig. 1 (and other figures as well) the colors do differ from ND (blue) and AD (red) treatment. However, PAR and T values being the same for both plots, this color code was not used. In addition, a horizontal line would help visually to differentiate the years. Does the referee mean a vertical line?

Fig 3: regression lines and $R^{2}$ (already given for $\mathrm{G} 14$ ) will be given also for MEGAN, but, in the ND plot, only for June and October $2012\left(0.25 x+7.7, R^{2}=0.32\right.$ and $1.4 x+0.45$, $R^{2}=0.82$ respectively), and April and June $2013\left(1.1 x+0.09, R^{2}=0.65\right.$ and $0.09 x+3.6$, $R^{2}=0.41$ respectively); in the AD plot only for April $2013\left(0.24 x-0.15, R^{2}=0.79\right.$ (see also reply to Referee\#2, Fig3). The month corresponding color is already given between the (a) and (b) graphs.

Fig. 5: \% is already mentioned; the legends of the $x$ axis is already given (on the top of the figure)

\section{Referee \#2}

We do thank Referee \#2 for her/his careful reading and useful comments/suggestions.

General comments

- Use of literature values instead of site-observed data: G14 was tuned using O3HP data, not literature data; this point being also mentioned by Referee \#3, it will be more clearly stated in the revised manuscript.

- No discussion of potential uncertainties from G14 and ANN in general: due to the overall words limitation we did not develop this important aspect of the work; a discussion will thus be added especially in the section 2.6 of the revised manuscript. Among the other available statistical methods, ANNs present the advantage of being the most 
parsimonious (e.g. giving the smallest error for a same number of descriptors; see for instance Dreyfus et al., 2002). Moreover, ANN approach, as the other non-linear regression methods, is not, or not very, sensitive to regressors' co-linearity (Bishop, 1995; Dreyfus et al., 2002). One of the ANNs limitations is that they can be used only for interpolation, not extrapolation exercises. For this reason, our future RCP projections we made using only $x i$ values that did fit into the range of variation of xi obtained during the training phase; in total $21 \%$ of data were thus rejected.

ANN optimization during the training phase was based on the reduction of the root mean square error (RMSE) between calculated and measured values. Our final optimized RMSE (validation data) was $8.5 \mu \mathrm{g} \mathrm{gDW}-1 \mathrm{~h}-1$ for our ER values ranging between 0.06 and $113 \mu \mathrm{g} \mathrm{gDW}-1 \mathrm{~h}-1$, and represents $35 \%$ of the mean $(22.7 \mu \mathrm{g} \mathrm{gDW}-1$ h-1).

- No comparison with process based model: as mentioned p3 in the first paragraph, p3, process based model require a complex set of data to be ran; such a dataset is not available for this study. This is the reason why an 'empirical' model (MEGAN) was solely tested.

- No conclusion and no clear key messages: a separate conclusion will be added in order to emphasize on: (i) the effect of a natural and amplified water stress on isoprene emissions from Q. pubescens (an increase of the emission factor), (ii) the difficulty of current empirical emission models to describe the isoprene emissions under stress conditions, (iii) the sensitivity of isoprene emission under water stress to soil water content, thermic stress and high frequency changes, on the site investigated.

- Abstract:

Too many abbreviations: all abbreviations used are explained in the abstract (as well as in the main paper) ; they were used in order to give the maximum information in the limited number of characters of the abstract.

Printer-friendly version

Discussion paper 
Few lines at the beginning to know why we need this study: we will add a sentence explaining that 'although strong isoprene emitters such as Mediterranean oaks are strongly affected by increasing drought and temperature, very little in situ information is available on how isoprene emissions will be impacted and how well the actual isoprene emission models can assess such impacts'.

- Method:

No description of MEGAN : indeed, MEGAN is generally not described when referred to, since it is widely used and known in the 'BVOC community'. A brief description with some important equations (see also Referee \#3 comment) and how we 'tuned' MEGAN in our study will be added in an new appendix.

No description on how ORCHIDEE data were used: predicted SW and ST were indeed assessed by running ORCHIDEE; as explained section 3.4 line 12, they were then used in the G14 algorithm in order to project/estimate isoprene emission under two future climatic scenarios. better describe how AD roof was operated: this point is also mentioned by Referee \#3; in the revised manuscript, we will explained that:

'A rainout-shelter above $300 \mathrm{~m}^{2}$ of canopy dynamically excludes precipitations (rain, snow and hail) since May 2012 by deploying automated shutters; the intercepted water was collected in a belowground reservoir which could be fed into an 'irrigated' control plot during times of exceptional drought periods (under construction). In the present study, the rainout shelter was deployed during rain events only from May to October 2012 in order to exclude $32 \%$ precipitation in the rain exclusion plot. Almost all the rains in late spring and summer were thus intercepted. Using ombrothermic diagram $\left(\mathrm{P}<2 \mathrm{~T}\right.$, with $\mathrm{P}=$ monthly precipitation in $\mathrm{mm}$, and $\mathrm{T}=$ monthly air temperature in $\left.{ }^{\circ} \mathrm{C}\right)$ we assessed that the summer 2012 drought period reaches 4.5 months in the AD plot, compared with 3 months in the ND plot. This percentage matches IPCC projections by the end of the year and fits the precipitation reduction at $\mathrm{O} 3 \mathrm{HP}$ during the driest years from 1967 to 2000 , compared to the average precipitation of this period (see figure I

Printer-friendly version

Discussion paper 
below).

Fig. I: Averaged cumulated precipitation at the O3HP between 1967 and 2000 (blue line with upper and lower standard deviation), and during the driest years of the same period (red line with upper and lower standard deviation). Cumulated precipitation in 2012 in the rain exclusion plot is represented by the green line and in the exclusion plot by the purple line.

\section{- Results:}

Use of on-site data to apply MEGAN rather than 'literature' data: this point being also raised by Referee\#3, a detailed - and common - answer is given in our reply to Referee\#3 ('Main concerns', last paragraph)

- Discussion:

Long text on future ER although no consideration on $\mathrm{C}$ source + training period for G14 is too short; $C$ source was indeed not directly considered since the hypothesis we made in applying a neuronal approach to statistically analyze our data was to consider 'simple' integrative environmental parameters; C source, nor vegetation adaptation, were thus not considered, but in the end we hypothesized that they are, more or less indirectly, driven anyway by PAR, T, SW and ST fluctuations over different range of frequencies. As vegetation adaptation is concerned, we did discuss this point in the manuscript in the end of section 4.3 ( $p$ 17) since we are aware that this aspect is of importance.

Thank you; we do agree that our database is not large enough to really assess impacts under future climates such as RCP2.6 and RCP8.5. In the revised manuscript, only RCP trends will be used in order to assess the sensitivity of our database to wilting point, $P$ decrease, $T$ increase, $P$ decrease $+T$ increase.

No conclusion : As mentioned in our reply to referee\#1, a proper conclusion will be added at the end of the manuscript

Printer-friendly version

Discussion paper 


\section{Detailed comments}

We agree with all other detailed comments / changes suggested but:

- P3, L5, References for empirical-based model: most of the empirical-based models use the same light and temperature dependences as those formulated by Guenther et al. (1995); currently, the most two widely used models are MEGAN (Guenther et al., 2006; 2012) and BEIS (Pier and Waldruff, 1991; Pierce et al., 1998). Emission estimates made using both models can often vary widely due to the differences in the land cover, emission factors and canopy models used. However models have not been found to outperform each other consistently when coupled to a air chemistry model and compared with measurements of some relevant atmospheric chemical species (e.g. ozone, aerosol, formaldehyde).

- P4, L3-8: indeed, the manuscript was structured in order to highlight the main scientific objectives of this study which were (i) to evaluate the in-situ impacts of ND and $A D$ on $Q$. pub, in order (ii) to develop a parameterization to represent ER variability using 'simple' integrated environmental parameters, and eventually (iii) to assess ER sensitivity to $\mathrm{P}$ decrease and/or $\mathrm{T}$ increase in the future.

- P5, L22: Only a small fraction ( $<5 \%)$ of the data was missing ; of course, the bias between Pcum curves at both sites was assessed and considered to 'extrapolated' the missing values at the O3HP site. As the precipitations were cumulated over 7, 14, 21 days, the bias was negligible (around 1\%).

- P6, L18: AF is dry leaf mass per area conversion factor, called LMA in a previous line. AF should hence be just changed by LMA.

We will check that all the abbreviations used are homogeneously cited.

Printer-friendly version

- P8, L1, How Krustal-Wallis test detects seasonal variation? Kruskal-Wallis tests allowed to check for different median values among months. ANOVA tests could not be used since data did not follow the requirements of parametrical tests. 
- P9, L31: we clarified earlier (fig a.) how the rain exclusion of $30 \%$ was achieved along the seasons.

- P10, L2-5: Although, in the absolute, the gap between Pcum(AD) and Pcum(ND) curves increases toward the end of 2012, from July 2012 the relative difference between both curves was maintained around $30 \%$ (fig. 1c: 510 and $760 \mathrm{~mm}$ respectively, thus a $33 \%$ reduction) .

- P11, L2, 'P=3.9' was a mistake.

- P11, L5-9: because the measurements were made right during the beginning of the isoprene onset period, some of the sampled branches started to emit isoprene significantly, while others emitted only at a very low level; this led to a large variability that could not be significantly related to the relative position of the branches in the AN or ND plots.

- P11, L13-14: these points were answered earlier in the General comments, section 'Results'

- P11, L18: indeed, as suggested by Referee \#1 (Detailed comments, Figure caption) the regression lines and $R^{2}$ will be added in Fig. $3 a$ and given in the text.

- P12, L6-7: our text was indeed not precise enough; we meant that under AD the contribution of the two lowest frequencies $(-14$ and $-21 \mathrm{~d}$.), was, RELATIVELY to the contribution of the two highest frequencies (instantaneous and -7d.), higher in April (48\%) and June (700 and $40 \%$ in 2012 and 2013 respectively) than during the summer (22, 8.5 and $16 \%$ in July, Aug. and Sept. respectively)'.

- P15, L21-24: the 'MEGAN adjustment' to our local data being also raised by Referee\#3, a detailed and common explanation is given in our reply to Referee\#3 (last paragraph of 'main concerns').

- P16, L12: although our revised manuscript will focus onto the sensitivity of isoprene to soil moisture rather than on 'large scale predictions', we do think it is important to 
remind, at least as a perspective in the new 'conclusion' section, that the reduction of the uncertainty on local/global isoprene emission assessments also require a better description of soil moisture model.

- Section 4.3: all result descriptions will be removed in order to tighten and focus this section onto discussion points only.

- P17: indeed, as mentioned in our answer to general comments, the revised manuscript will present sensitivity tests of ER to T, Pcum and SW, rather than future projections; because there is no other in situ long term study of drought effect on isoprene emissions, direct comparisons are not possible; however we will give more details on the findings obtained during a seasonal study carried out on isoprene emission from Q. pub. saplings by Genard-Zielinski et al. (2014) - already cited in our manuscript. We will then extend our discussion and compare our results to other in situ drought study in the Mediterranean area, but not for isoprene. For instance, Lavoir et al. (2009) reported lower monoterpene emission rates from $Q$. ilex in the rain exclusion plot from June to August, during the 2 nd and 3rd year or rain exclusion. Since $Q$. ilex does not possess specific leaf reservoirs for monoterpene storage, emissions of $Q$. ilex respond to light and temperature as isoprene emissions. Their emission is hence de novo and tightly related to their synthesis. Monoterpene emissions of this species are thereby comparable to isoprene emissions.

- Figure1: we will add the $x$ axis legend also on the top of the (a) graph in order to make the reading easier

- Figure 2: Indeed, differences between ND and AD using Mann-Whitney tests should be denoted with asterisks at the top of each figure. Differences among months were tested using Kruskal Wallis tests (W) followed by followed by Newman-Kruels post-hoc test with $\mathrm{a}<\mathrm{b}<\mathrm{c}<\mathrm{d}$. These corrections will be made.

Note that the Gw unit should be "mmol cm-2 s-1" and not "mol cm-2 s-1". 
- Figure 3: unfortunately log scale was not suitable for this figure since many calculated values was set to zero by MEGAN; here after we present the log/log figures with the remaining non zero values (Fig. II)

- Figure 5: indeed, the '\%T' and '\%W' abbreviations are missing, as well as 'inst', '7d', '14d' and '21d'.

\section{Referee \#3}

We do thank Referee \#3 for her/his careful reading and useful comments and suggestions.

In order to make the reading smoother and the understanding clearer, the revised version of the manuscript will be proofread by a native English speaker before submitted a revised version. In addition, a special attention will be paid to better link the development of the different discussions.

\section{Main concerns:}

- Bibliographical weakness:

Although the references cited are not all 'narrow in focus and scope' and that a large number of references given do already focus on the effects of drought onto photosynthesis/cellular processes/isoprene emissions (Chaves et al., 2002; Lichtenthaler et al., 1997; Funk et al., 2004, Simon et al., 2005; Tani et al., 2011; Guenther et al., 2013; Wiberley et al., 2005; Llusià et al., 2008, 2009; Owen et al., 1998; Loreto \& Schnitzler, 2010; Pegoraro et al., 2004) we will also cite the works of Sharkey and Loreto (1993) and Brili et al (2007).

Note that we already referred twice to the Peñuelas and Staudt (2010) paper (in the Introduction p2 and in the Discussion p16), which is a review, and thus, inherently, cannot be considered as narrow.

Although our manuscript do not aim at reviewing all the isoprene modelling studies 
based on MEGAN, the excellent work of Müller et al. (2008) will be additionally considered and their assessments of the sensitivity of isoprene emissions to soil moisture will be presented; note however that Müller et al. (2008) reported that, when MEGAN model is evaluated against the 2 forest sites of Harvard (US) and Tapajos (Amazonia), it does 'fail to reproduce the observed seasonal variation at the tropical rainforest site' (wet and dry seasons).

The assessment of the wilting point teta(w) in the Sindelarova et al., 2014 study was based in the Pegoraro et al. (2004) work that we do already refer to in our discussion: as mentioned in our manuscript, Sindelarova et al. (2014) remind that under this threshold value, the isoprene emission is set to zero.

As we did observe isoprene emissions when soil moisture values were as low as 0.007 m3 m-3, we considered that testing the sensitivity of ER to teta(w) using so low values was pointless and not relevent regarding the soil type present at the O3HP; we did conclude that the Pegoraro approach was not valid for drought adapted tree species.

Although we did not mention it, we did assess the wilting point teta(w) at the $03 \mathrm{HP}$ to be $0.15 \mathrm{~m} 3 \mathrm{~m}-3$, a value very close to the $0.138 \mathrm{~m} 3 \mathrm{~m}-3$ value (Chen et Dudhia, 2001) we used; since our original manuscript was aiming at producing large scale projections, we made the choice to test MEGAN tuned with this teta(w) literrature data. Anyway, this slight difference of wilting point value does not change, in the end, the performance of MEGAN which cannot represent isoprene emissions for SW conditions much smaller thant the teta $(\mathrm{w})$ value (MOST of the calculated ER remained set to zero).

Only when the SW effect was turned off in MEGAN (set to 1 for every calculation), were we able to test the effect of using on site isoprene emission factors Is rather than the Simpson et al. (1999) one (53 $\mu \mathrm{gC} \mathrm{gDW-1} \mathrm{h-1);} \mathrm{we} \mathrm{present} \mathrm{and} \mathrm{discuss} \mathrm{later} \mathrm{the}$ results of such a comparison (see Fig III and corresponding text).

- Measurements:

Printer-friendly version

Discussion paper 
1. We will make it clearer in the revised manuscript but :

- we did not aim at extrapolating - using MEGAN or another emission model - isoprene fluxes from the whole canopy; our focus was to study, for the first time in situ and over such a time scale, the ND and AD drought impacts on (only) sunlit branches of Q. pub; however some measurements were made under the canopy (see later on).

- thus, all the primary production, stomatal conductance and isoprene emission rates presented in this study were - only - measured for the leaves sampled at the top of the canopy ; the effect of the canopy structure is not addressed in this paper.

- the shading effect was investigated, only for 2 trees in the ND plot, and only in June 2012; we observed that, although the shading effect can be strong (PAR lowered by a factor of 6 to 18 ), measured isoprene emission factors (Is) were not significantly different $(\mathrm{P}>0.05)$ at the top and below the canopy $(77+/-3$ and $59+/-12 \mu \mathrm{g}$ gDM-1 $\mathrm{h}-1$ respectively); these points are detailed in another paper (Genard-Zielinski et al., 2015).

2. As noticed by Referee \#2 our manuscript is lacking of clear details on how the roof was operated in order to simulate an AD. More information is given in our reply to Referee\#2. Figure I shows that, during this study, the $30 \%$ precipitation reduction was reached quickly after the operation of the roof operation started, in May 2012.

When this study was conducted we were well aware that the future climatic changes will affect not only the intensity of the precipitations but the period over which these precipitations will occur. Since our study started just after the roof operation was available; we did focus, for this 1st year of measurements, only on the exclusion intensity aspect rather than its 'timing'.

3. We don't see any scientific basis for which Referee\#3 could believe that we do relate the reality of our work. So, yes, a tremendous effort was made at the O3HP to conduct our field work and YES, we did sample Q. pubescens over one week per every month, as detailed in the Table below. Moreover we did not state that we sampled

Printer-friendly version

Discussion paper 
isoprene 'one week per month from June 2012 till June 2013'; we did precise that 'measurements were performed at least during one week, once a month, from June 2012 to June 2013, except from November 2012 to March 2013 when Q. pubescent is fully senescent with leaves remaining on the tree' (P7, L26-28).

June $2012: 1$ - 16;

July 2012: 15 - 20;

August 2012:19 - 24;

September 2012:18 - 24;

October 2012: 22 - 26;

April 2013 : 22 - 27; June 2013: 16 - 22;

All isoprene measurements presented here were sampled on cartridges except during April 2013 where only on-line PTRMS was used (and no cartridges). An intercomparison between Cartridge+GCMS and PTRMS was carried on parallel on another emitter present on the site (Acer monspessulanum); no significant difference was observed between both techniques (see Genard-Zielinski, 2014 for details).

As mentioned earlier, this seasonal study was not planned to focus on vertical profile of BVOC through the canopy.

\section{The COOPERATE data:}

All the data (PAR, ambient T, Precipitation, SW, ST) used to train the ANN were obtained from the COOPERATE dataset; we used daily averaged values. This information will be added in the revised manuscript.

As explained earlier to referee\#2, only a very few number $(<5 \%)$ of precipitation data needed for assessing $P$ integrated over 7, 14 or 21 days, was missing. As we spent quite a lot of time on the site, we are well aware that precipitations can, locally, be 
highly variable in time and space; this is why we used the relative differences observed between the 2012 and 2013 Pcum curves in both sites (Forcalquier and O3HP) in order to 'fill the gap' of the missing data. Due to the fact that the $\mathrm{P}$ was integrated over 7 , 14,21 days, we assumed that this bias remains negligible compared to reducing the number of data in our ANN study due to missing $P$ values.

\section{Statistics}

1. We are aware on how the 'G91' and 'G13' parameterizations lead to the integrated and much more complex emission model MEGAN; we know that MEGAN does include some 'historical' dependence of environmental conditions as we - too briefly mentioned and discussed P15, L19-20. Thus, of course, we did consider the PAR and T over the previous 24 and 240 hours before our measurements in our calculated isoprene emissions (as we did consider and applied the leaf age dependence gamma(age), from eq(2) page 3189, Guenther et al., 2006)). We agree that these points should be more clearly stated. Moreover, we should more clearly remind that MEGAN does consider some 'historical' effect.

As we did not consider the canopy effect in our study, the canopy structure dependence as considered in MEGAN through the gamma(CE) parameter (from eq(2) page 3189, Guenther et al., 2006) was not considered (i.e. set to 1).

As we measured emission rates using a chamber but not canopy fluxes, we did not have to consider any canopy loss (we set gamma=1, from eq(1), page 3183, Guenther et al., 2006).

In the revised manuscript, and as also suggested by Referre\#2, we shall more clearly detail these important aspects to help the reader to better understand how we tuned MEGAN for assessing our emission rates. We shall also emphasize our knowledge that MEGAN does account for the 'history' of the plant, better than we did on P15, L19-20. 
As also requested by Referee\#2 the CL CT parameterizations and a brief description of MEGAN will, thus, be presented in a new appendix.

2. We agree that our dataset is neither large enough nor robust enough for capturing future emission under RCP scenarios. As mentioned earlier and as suggested by Referee\#2, the use of $\mathrm{G} 14$ will be limited in the revised version, to some sensitivity tests to temperature and/or drought changes.

3. Indeed ANN is often referred to as a 'grey' box, since such a statistical approach does not aim at providing some new mechanistic understanding of the studied process. On the other hand, when a mechanistic approach is failing to do so, ANN can provide some fruitful directions to where the 'mechanistic approaches' shall be looking at. In our case, some knowledge was gained by demonstrating, in particular, that the dependence of isoprene emissions under ND and AD (at least for Q. pub.):

- should be considered on a frequency lower than 10 days (as in MEGAN) and at least up to 3 weeks, and not only for $L$ or $T$ parameters

- are related to ST (soil temperature) as well, highlighting - probably - the possible impact of the soil microorganism functioning on isoprene emissions

- cannot be assessed, for drought adapted plant, by a 'threshold' type approach such as in MEGAN.

Eventually, note that in MEGAN, the isoprene dependency to PPFD over the previous 24 and 240h was deduced by a 'best statistical fit' (P3190, L28-32 Guenther et al., 2006), without providing any precise mechanistic insight on how and why it is working; the useful of MEGAN is, nevertheless, not challenged.

Concerning the use of site-specific data, we already answered and discussed earlier to this point; the figures III below present the effects of using our on-site isoprene emission factors Is rather than the value of 53 given by Simpson et al. (1999), WHEN on only WHEN no SW effect was considered in MEGAN. We observe that :

Printer-friendly version

Discussion paper 
- in the control plot (ND), less variability was represented by MEGAN when on-site Is were used (fig c) compared to Is=53 (fig a) (39\% compared to $46 \%$ respectively) and that the overall underestimation was reduced (from 47 to $26 \%$ respectively)

- in the stress plot (AD): only $40 \%$ of the variability was represented by MEGAN, whatever the Is used; an over-estimation of $18 \%$ was obtained when on-site Is were used (fig d) compared to an underestimation of more than a factor 2 when Is was set to 53 (fig b). Although these results do not represent the full architecture of MEGAN (SW effect was not activated) they could be presented and discussed in the revised manuscript.

\section{ORCHIDEE}

As explained P9, L8, ORCHIDEE was run to assessed SW and ST for the RCP exercises.

As explained P8, I26 to P9 L6, ORCHIDEE used ISI-MIP met data for RCP projections.

Our projections were not made specifically for the O3HP site, but for a Mediterranean site representative of the O3HP site conditions. Therefore, precipitations -or other parameters - were not downscaled to the O3HP site; they are representative of the whole cell which corresponds to the one where the O3HP site is located.

\section{Recommendations}

Site-specific data: this point is discussed several times above.

We remind once more that the wilting point was assessed for the O3HP site; when used, MEGAN does not agree better with our observations.

As explained earlier, physiological parameters such as sap flow, transpiration, ... were not measured during this work; our study aimed at relating our observations to 'more simple', more 'integrative' environmental variable, 'easily' accessible than complex physiological data; we hypothesized that, anyway, sap flow, transpiration, ... are in the end, more or less indirectly, driven by L, T, SW, ST considered over a large range

Printer-friendly version

Discussion paper 
of time.

Detailed comments

None

New references cited in our responses

Sharkey, T. D. and Loreto, F.: Water stress, temperature, and light effects on the capacity for isoprene emission and photosynthesis of kudzu leaves, Oecologia, 95, 328-333, 1993.

Brilli, F., Barta, C., Fortunati, A., Lerdau, M., Loreto, F., and Centritto, M.: Response of isoprene emission and carbon metabolism to drought in white poplar (Populus alba) saplings, New Phytol., 175, 244-254, 2007.

A.-V. Lavoir1, M. Staudt1, J. P. Schnitzler2, D. Landais1, F. Massol3, A. Rocheteau4, R. Rodriguez1, I. Zimmer2, and S. Rambal1, Drought reduced monoterpene emissions from the evergreen Mediterranean oak Quercus ilex: results from a through fall displacement experiment, Biogeosciences, 6, 1167-1180, 2009

Please also note the supplement to this comment:

https://www.biogeosciences-discuss.net/bg-2017-17/bg-2017-17-AC1-supplement.pdf

Interactive comment on Biogeosciences Discuss., https://doi.org/10.5194/bg-2017-17, 2017. 


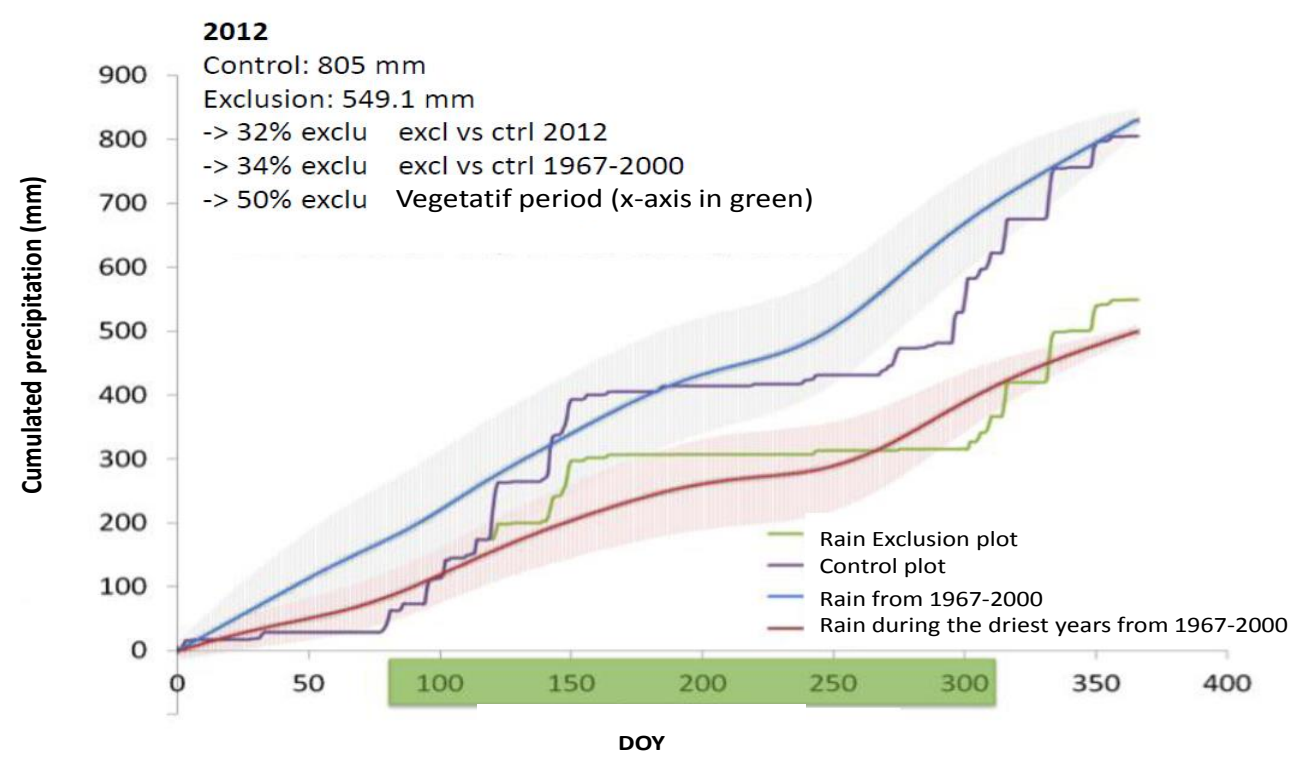

Interactive

comment

Fig. I: Averaged cumulated precipitation at the $\mathrm{O}_{3} \mathrm{HP}$ between 1967 and 2000 (blue line with upper and lower standard deviation), and during the driest years of the same period (red line with upper and lower standard deviation). Cumulated precipitation in 2012 in the rain exclusion plot is represented by the green line and in the exclusion plot by the purple line.

Fig. 1. 


\section{Figure II}
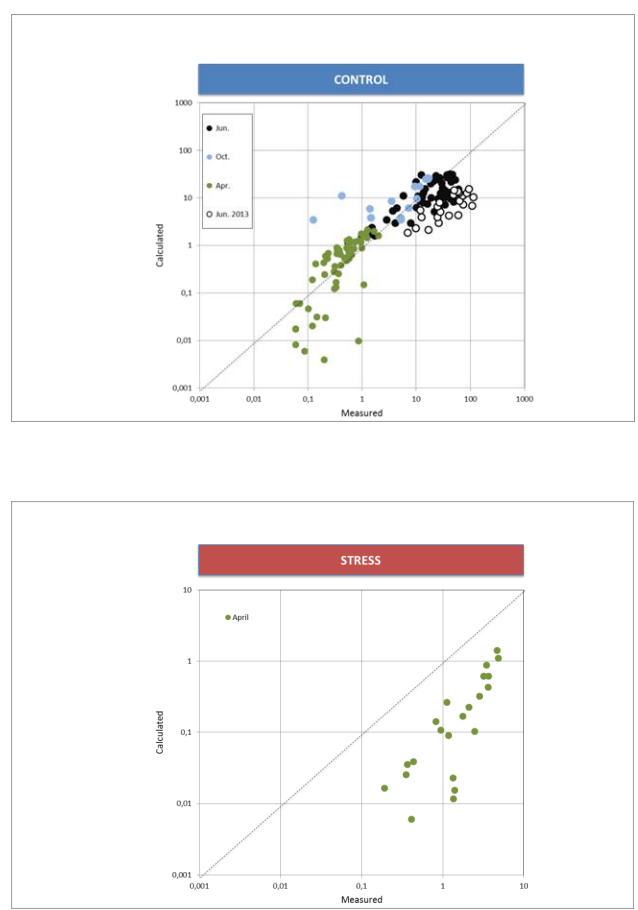

Printer-friendly version

Fig. 2.

Discussion paper 


\section{BGD}

Figure III
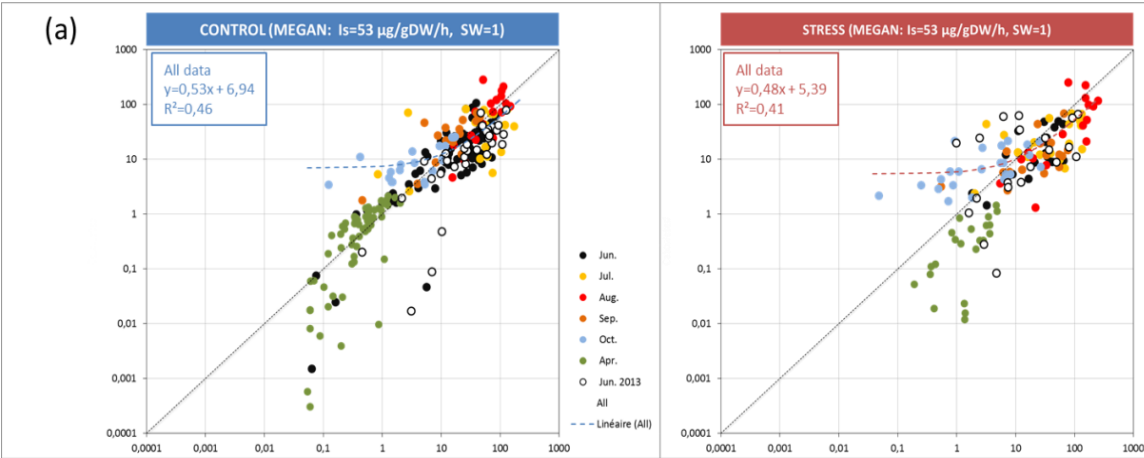

Interactive

comment
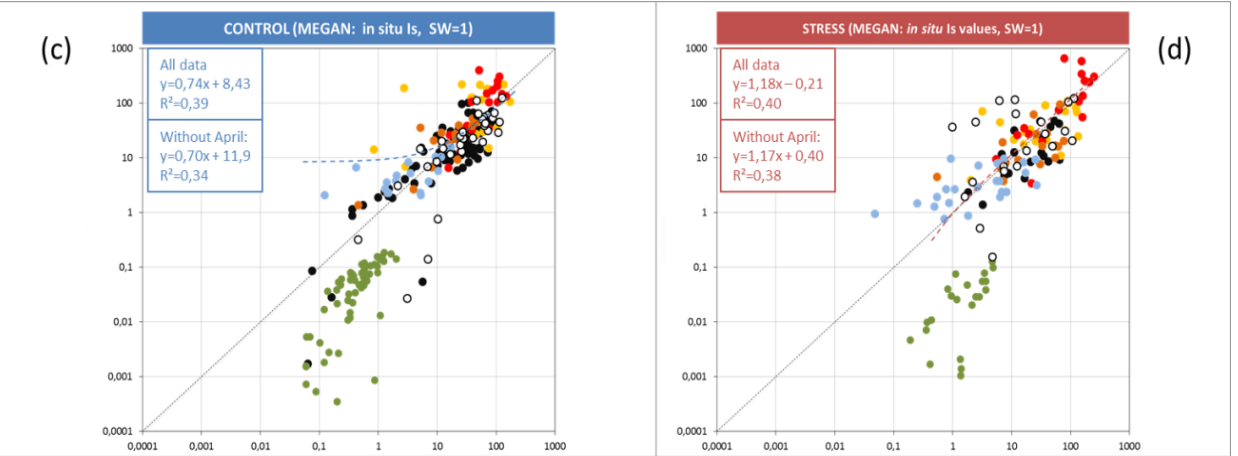

Fig. 3. 